\title{
Effect of One Percent Chlorhexidine Addition on the Antibacterial Activity and Mechanical Properties of Sealants: An in vitro Study
}

\author{
${ }^{1}$ Arunachalam Karthikeyan Shanmugaavel, ${ }^{2}$ Sharath Asokan, ${ }^{3} \mathrm{~J}$ Baby John, ${ }^{4}$ PR Geetha Priya, ${ }^{5}$ Jagadeesan Gnana Devi
}

\begin{abstract}
Aim: The aim of the study was to evaluate the effect of addition of $1 \%$ chlorhexidine digluconate solution on the antibacterial activity and mechanical properties of glass ionomer and resin based sealant.
\end{abstract}

Materials and methods: Conventional glass ionomer sealant (GIS) (Fuji VII, Japan) and resin sealant (Clinpro 3M ESPE, USA) were used in this study. Chlorhexidine digluconate (CHX) (20\%) liquid was added to both the sealants, and the concentration of chlorhexidine in sealants was adjusted to $1 \%$. The sealants were divided into four groups as: group A (GIS), group $B(\mathrm{GIS}+1 \% \mathrm{CHX})$, group $\mathrm{C}$ (resin sealant), group $\mathrm{D}$ (resin sealant $+1 \% \mathrm{CHX}$ ). Five cylindrical specimens were prepared in each group. Their antibacterial activity against Streptococcus mutans and Lactobacillus acidophilus, and their mechanical properties (compressive strength and diametrical tensile strength) were assessed. Mann-Whitney and Wilcoxon signed rank test were used appropriately for statistical analysis (SPSS version 19).

Result: Addition of one percent chlorhexidine significantly increased the antibacterial activity of both the sealants. There was a significant difference between groups $A$ and $B(p<0.009)$, and groups $C$ and $D(p<0.008)$. There was no significant difference in the mechanical properties of the sealants.

Conclusion: Addition of one percent chlorhexidine to the glass ionomer and resin based sealants provided sufficient antibacterial activity, without significantly affecting the mechanical property of the sealants.

Keywords: Antibacterial activity, Chlorhexidine, Pit and fissure sealants.

${ }^{1}$ Postgraduate Student, ${ }^{2}$ Professor, ${ }^{3}$ Professor and Head

${ }^{4}$ Reader, ${ }^{5}$ Dental Surgeon

${ }^{1}$ Department of Pedodontics and Preventive Dentistry, KSR Institute of Dental Science and Research, Tiruchengode, Tamil Nadu, India

${ }^{2-4}$ Department of Pediatric Dentistry, KSR Institute of Dental Science and Research, Tiruchengode, Tamil Nadu, India

${ }^{5}$ Department of Dentistry, Arunz Dental Clinic, Chennai, Tamil Nadu, India

Corresponding Author: Arunachalam Karthikeyan Shanmugaavel, Postgraduate Student, Department of Pedodontics and Preventive Dentistry, KSR Institute of Dental Science and Research, Tiruchengode, Tamil Nadu, India, Phone: 9941578986 , e-mail: dentistshan@gmail.com
How to cite this article: Shanmugaavel AK, Asokan S, John JB, Geetha Priya PR, Gnana Devi J. Effect of one percent Chlorhexidine Addition on the Antibacterial Activity and Mechanical Properties of Sealants: An in vitro Study. Int J Clin Pediatr Dent 2015;8(3):196-201.

Source of support: Nil

Conflict of interest: None

\section{INTRODUCTION}

'An ounce of prevention is better than a pound of cureBenjamin Franklin'. Dental caries, second to common cold, remains one of the most prevalent chronic diseases that affect children and young adults globally. The world is now aiming to prevent this disease at an early stage and provide children the best quality of life. Pit and fissure sealants provide both primary and secondary levels of prevention by preventing caries initiation and progression. ${ }^{1}$ Microleakage around the sealants provide pathway for the bacteria and initiate secondary caries. If these caries lesions progress, they may eventually result in pain and pulp exposure in young children.

An antibacterial agent is a substance that either kills bacteria or inhibits their growth. Chlorhexidine digluconate is a cationic biguanide which has gained attention for its antibacterial properties. Jedrychowski (1983) was the first to report the antibacterial property of the restorative material after the addition of chlorhexidine. ${ }^{2}$ Many studies have been carried out by adding chlorhexidine to glass ionomer cement, but there were limited studies stating the addition of chlorhexidine to resin materials. ${ }^{2-6}$ Adding antibacterial activity to the sealants strengthens the defense mechanism of the later against the bacteria. Hence, this study was planned to evaluate the antibacterial activity and mechanical properties of the glass ionomer and resin based sealants after the addition of $1 \%$ chlorhexidine digluconate.

\section{MATERIALS AND METHODS}

The study protocol was analyzed and approved by institutional review board of KSR institute of dental science and research, Tiruchengode, India. Conventional glass ionomer sealants (GIS) (Fuji VII, Japan) and resin sealants 
(Clinpro 3M ESPE) were used in this study. Test specimens were prepared by adding $20 \%$ chlorhexidine digluconate (CHX) liquid (Anabond Stedman pharma research, Chennai, India) in the following proportions: $0.05 \mathrm{ml}$ of the CHX was pipetted and added to $0.95 \mathrm{ml}$ of the liquid portion of the GIS and to the $0.95 \mathrm{ml}$ of resin sealant to adjust the concentration to $1 \% \mathrm{CHX}(\mathrm{w} / \mathrm{w})$. The sealants were divided into four groups namely: group $\mathrm{A}$ (GIS), group B (GIS + 1\% CHX), group C (resin sealant), group $\mathrm{D}$ (resin sealant $+1 \% \mathrm{CHX}$ ). Five specimens were prepared in each group. The antibacterial activity was assessed via agar diffusion test against Streptococcus mutans and Lactobacillus acidophilus in the department of biotechnology (KSR group of institutions, Thiruchengode, Tamil Nadu, India), and the mechanical properties (compressive strength and diametrical tensile strength) were examined at central institute of plastic engineering and technology, Chennai.

\section{Agar Diffusion Test}

Streptococcus mutans and L. acidophilus are the most common pathogens involved in the initiation and progression of the carious lesion. These bacteria were obtained from microbial type culture collection and gene bank, Chandigarh, India. The antibacterial activity of the set material against S. mutans (MTCC 497; microbial type cell culture, Chandigarh) and L. acidophilus (MTCC 10307; microbial type cell culture, Chandigarh) was assessed using agar diffusion test. All the procedures were done in an aseptic laminar cabinet.

The strains of L. acidophilus and S. mutans, stored in $50 \%$ glycerol at $-20^{\circ} \mathrm{C}$ were cultivated in brain heart infusion (BHI) and Lactobacillus MRS agar (Hi Media Laboratories Pvt. Ltd, Mumbai, India) broth, respectively at $37^{\circ} \mathrm{C}$. A loopful of inoculum was transferred to $10 \mathrm{ml}$ of BHI and Lactobacillus MRS agar broths after 48 hours incubation. Five wells of $6 \mathrm{~mm}$ diameter were cut using a well cutter in commercially available Muller Hinton agar (MHA) and Mullen Hinton agar supplied with 5\% sheep blood (Bioline laboratory, Coimbatore, India) agar plates of $90 \mathrm{~mm}$ diameter and $4 \mathrm{~mm}$ thickness. Bacterial suspensions of $350 \mu \mathrm{l}$ of L. acidophilus and S. mutans were streaked over the agar plates.

The GIS were mixed according to powder/liquid ratio of 1:1 with sterile spatula on a mixing pad and placed into wells within 1 minute after mixing. Similarly, the resin based sealants were placed into the wells and cured with halogen light curing unit (Elspar 2500, 3MESPE, USA) with light intensity of $410 \mathrm{~mW} / \mathrm{cm}^{2}$, on both sides to ensure complete curing. ${ }^{4}$ Plain $20 \% \mathrm{CHX}$ digluconate was used as positive control (E). The agar plates were kept at room temperature for 2 hours for diffusion of the material into the medium and incubated at $37^{\circ} \mathrm{C}$ for 48 hours. Zones of inhibition at day 0 were measured by a single calibrated examiner. The zones of inhibition were measured by subtracting the well diameter from the average zone of inhibition measured at three different points using digital vernier calipers (Aerospace, China) in millimeters $(\mathrm{mm})$. The specimens were incubated for another 5 days at $37^{\circ} \mathrm{C}$ and then fresh subcultures were made; the procedure was repeated to measure the zones of inhibition after the 7th day. The antibacterial activity was measured again after 30 days by transferring the specimens at 23rd day and incubating for 48 hours in fresh inoculated plates. ${ }^{4}$

\section{Mechanical Property}

Plastic molds were prepared according to American dental association (ADA) no. 27, of diameter $4 \mathrm{~mm}$ and height $6 \mathrm{~mm}$. The GIS sealants were mixed as mentioned above and placed into a cylindrical plastic mold and covered with glass plates. ${ }^{4}$ The resin sealants were placed in the plastic mold in $2 \mathrm{~mm}$ increment for three times and cured with halogen light curing unit (Elspar 2500, 3MESPE, USA) with light intensity of $410 \mathrm{~mW} / \mathrm{cm}^{2}$ on both sides to ensure complete curing. The materials were allowed to set for 30 minutes. The plastic molds were then removed and checked for the presence of voids. Specimens with voids were discarded. The cylindrical specimens were finished using carborundum disks and stored in distilled water for 24 hours. ${ }^{4}$ Prior to testing, the diameter of the specimens were determined using a micrometer gauge.

The specimens were then subjected to universal testing machine (Shimadzu, Japan). Compressive strength (CS) of the material was tested by giving load along the long axis of the specimen with cross head speed of $1 \mathrm{~mm} / \mathrm{min}$ until fracture of the specimen occurs. ${ }^{4}$ The value of the force (F) measured in Newtons and diameter (d) was applied in the formula $C S=(4 \mathrm{~F}) /\left(\pi \mathrm{d}^{2}\right)$, to find out the compressive strength value in Megapascal (MPa).

Diametrical tensile strength (DTS) was measured by directing the force against the lateral surface of the specimen with cross head speed of $1 \mathrm{~mm} / \mathrm{min}$ until fracture occurs. ${ }^{4}$ The value of the force (F) measured in Newtons and diameter (D) was applied in the formula $\mathrm{DTS}=(2 \mathrm{~F}) /(\pi \mathrm{DT})$, to find out the DTS value in Mega pascal (MPa).

The results were tabulated and statistically analyzed using SPSS version 19.0 software (SPSS Inc., Chicago Ill, USA). Mann-Whitney U test and Wilcoxon signed rank test were used appropriately for intergroup and intragroup comparisons. A p-value $\leq 0.05$ was considered statistically significant. 


\section{RESULTS}

\section{Agar Diffusion Test}

Table 1 shows the results of agar diffusion test against S. mutans and L. acidophilus after 0, 7 and 30 days. Figures $1 \mathrm{~A}$ to $\mathrm{F}$ depict the zones of inhibition for $L$. acidophilus and S. mutans. There was a significant increase in the antibacterial activity of the sealants against $S$. mutans [group B $(\mathrm{p}<0.009)$; group $\mathrm{D}(\mathrm{p}<0.008)$ ] and L. acidophilus [group B $(\mathrm{p}<0.009)$; group D $(\mathrm{p}<0.008)$ ] immediately (day 0 ) after the addition of $1 \%$ chlorhexidine. This antibacterial activity decreased statistically after 7 days against $S$. mutans [group B $(p<0.042)$, group D $(p<0.042)$ ] and L. acidophilus [group B ( $\mathrm{p}<0.041)$ ]; while it was not statistically significant (NS) in group $\mathrm{D}(\mathrm{p}<0.068)$. This antibacterial activity continued to exist until 30 days. But no antibacterial activity was seen in conventional GIS and resin sealants (groups A and C) after 7 and 30 days.
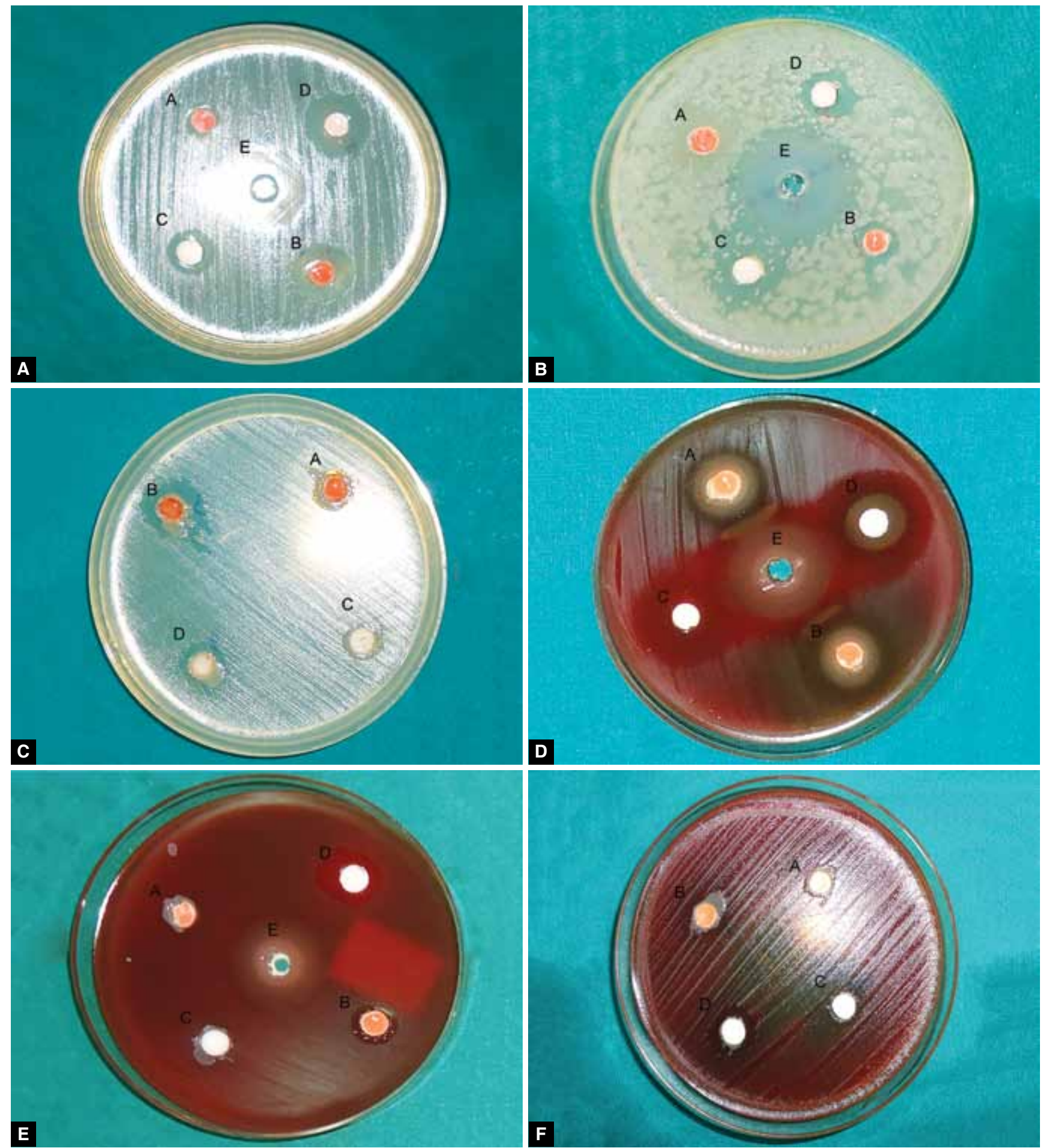

Figs 1A to F: (A to C) Zones of inhibition in various groups against $L$. acidophilus on day $0,7,30$ and (D to $F$ ) Zones of inhibition in various groups against S. mutans on day 0, 7, 30 (A: Glass ionomer sealant; B: GIS + 1\% CHX; C: Resin sealant; D: Resin sealant + $1 \% \mathrm{CHX}$; $\mathrm{E}: 20 \% \mathrm{CHX}$ ) 
Table 1: Comparison of the mean zones of inhibition $(\mathrm{mm})$ against $S$. mutans and $L$. acidophilus among the four groups at different time periods

\begin{tabular}{llllllll}
\hline & \multicolumn{3}{c}{ S. mutans } & & \multicolumn{3}{c}{ L. acidophilus } \\
\cline { 2 - 4 } \cline { 6 - 8 } Groups & 0 day & 7 days & 30 days & & 0 day & 7 days & 30 days \\
\hline A & 7.28 & 0 & 0 & & 4.16 & 0 & 0 \\
B & 10.36 & 5.7 & 1.67 & & 9.7 & 5.02 & 1.5 \\
C & 11.96 & 0 & 0 & & 4.4 & 0 & 0 \\
D & 14.82 & 8.68 & 5.83 & & 10.18 & 8.16 & 4.83 \\
\hline
\end{tabular}

\section{Mechanical Property}

Table 2 shows that the compressive and diametrical tensile strengths of the sealants were not altered significantly after the addition of $1 \%$ chlorhexidine.

\section{DISCUSSION}

By any standards, advancements toward caries prevention have been impressive during the past few decades. Pit and fissures are eight times more susceptible than smooth surface to caries. ${ }^{7}$ Any tooth judged for caries risk can certainly benefit from sealant application, which acts as a physical barrier to microorganism and other food particles. Achieving a perfect isolation in young children is a very tough task. Placement of sealants is a very sensitive technique and is influenced by several factors, such as patient cooperation, contamination of the operating field and operator variability. ${ }^{8}$ The penetration of sealants in pits and fissures also depends on their. ${ }^{9}$ These factors can lead to microleakage, plaque accumulation around restorations or loss of restoration, which results in secondary caries. Secondary caries has two types of lesion namely: (A) outer lesion which occurs as a result of plaque accumulation, and (B) inner lesion which occurs due to microleakage between the tooth and restoration. Microleakage occurs around the sealants irrespective of the preparation methods and provides a pathway for the food particles, oral fluids and microorganisms to penetrate in the gaps, and initiate secondary caries. ${ }^{10}$ An ideal system with antibacterial activity reduces plaque accumulation on or near surfaces, and the number of microorganisms in oral cavity, thereby eliminating secondary caries. ${ }^{3}$

Table 2: Comparison of the mechanical properties among the four groups

\begin{tabular}{|c|c|c|c|c|}
\hline \multirow[b]{2}{*}{ Groups } & \multicolumn{2}{|c|}{$\begin{array}{l}\text { Compressive } \\
\text { strength }\end{array}$} & \multicolumn{2}{|c|}{$\begin{array}{c}\text { Diametrical tensile } \\
\text { strength }\end{array}$} \\
\hline & $\begin{array}{l}\text { Mean } \pm S D^{*} \\
(M P a) \dagger\end{array}$ & $p$-value & $\begin{array}{l}\text { Mean } \pm S D^{*} \\
(M P a) \dagger\end{array}$ & $p$-value \\
\hline$A$ & $17.10 \pm 9.07$ & $0.95 \mathrm{NS}$ & $6.5 \pm 1.32$ & $0.52 \mathrm{NS}$ \\
\hline B & $16.82 \pm 4.63$ & & $5.51 \pm 1.57$ & \\
\hline C & $123.54 \pm 47.68$ & $0.60 \mathrm{NS}$ & $43.37 \pm 13.77$ & $0.58 \mathrm{NS}$ \\
\hline D & $107.93 \pm 42.06$ & & $32.69 \pm 21.76$ & \\
\hline
\end{tabular}

Chlorhexidine, a cationic polyguanide has been primarily used as disinfectant. The bactericidal effect is the result of this cationic molecule binding to the negatively charged bacterial cell walls. It is bacteriostatic and bactericidal at low and high concentrations respectively.

McCue et al (1951) have reported that quick setting acrylic has slight antibacterial activity. ${ }^{11}$ Its addition in mouthwashes has reduced plaque accumulation and hence helped in the maintenance of good oral hygiene. Since 1980, CHX addition has been tried in various forms and percentages in glass ionomer cement and composite resin to provide antibacterial activity. Chlorhexidine can be added to restorative materials either as powder or liquid, in percentages ranging from 1 to $10 \% .^{2-5}$ Jedrychowski et al (1983) suggested that chlorhexidine added as powder form can be a better option than the liquid form. ${ }^{2}$ The antibacterial activity increased proportionately as the concentration of the liquid CHX increased but the mechanical properties were altered significantly in concentrations greater than $1 \% .{ }^{2,4}$ Hence, in this study $1 \%$ chlorhexidine digluconate liquid was added to the sealants. After $\mathrm{CHX}$ addition to resin sealants, it was stirred well with a sterile glass stirrer and the test specimens were prepared immediately. Agar plate diffusion was used in this study because it allowed analyzing both set and unset materials. ${ }^{12}$ This study was an attempt to add chlorhexidine to the sealants to enhance their antibacterial activity and make them more effective in prevention.

The differences in the zones of inhibition for L. acidophilus and S. mutans at three different time intervals are shown in Graphs 1A and 1B respectively. Results after day 0 showed antibacterial activity in all the groups, while no antibacterial activity was observed in groups A and $C$ after 7 days and 30 days. This was in accordance with results shown by Naoungroj et al (2010) that conventional sealants provide surface antibacterial activity as a

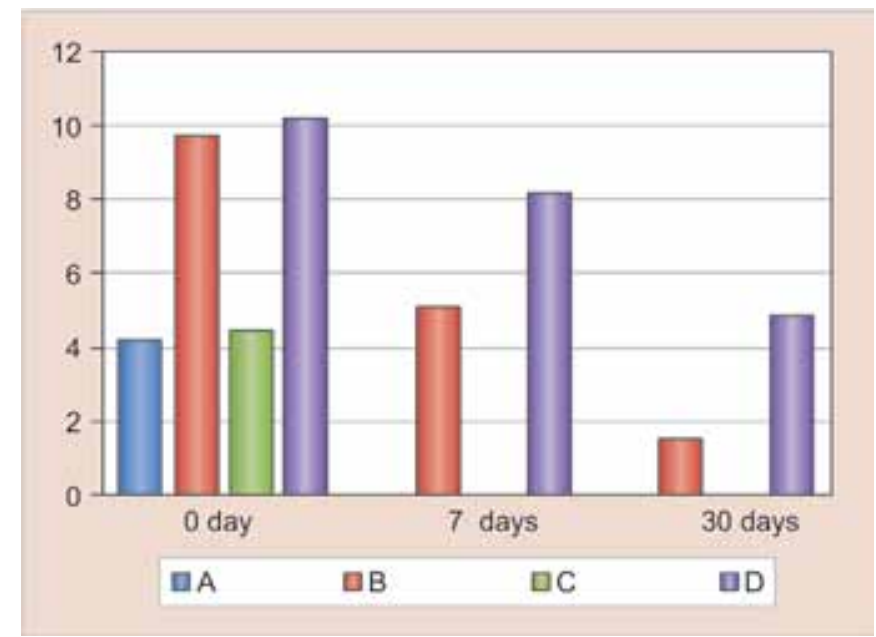

Graph 1A: Comparison of mean scores of zones of inhibition of various groups against $L$. acidophilus on day $0,7,30$ 


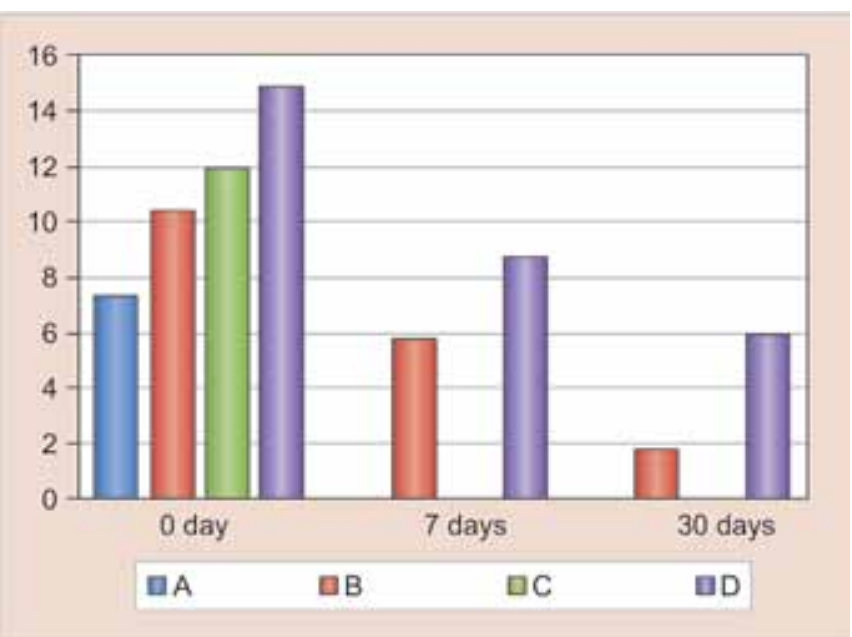

Graph 1B: Comparison of mean scores of zones of inhibition of various groups against $S$. mutans on day 0,7,30; (A: Glass ionomer sealant; B: GIS + 1\% CHX; C: Resin sealant, D: Resin sealant + $1 \% \mathrm{CHX}$

result of fluoride release. ${ }^{13}$ Matalon et al (2010) showed that sealants aged 30 days showed no antibacterial activity. ${ }^{14}$ Specimens in groups B and D showed antibacterial activity even after a period of 30 days. Similar results were shown by Turkun et al (2008) in their study with antibacterial activity lasting for 70 days in GIC with $1.25 \%$ chlorhexidine digluconate. ${ }^{4}$ Takemura et al (1983) reported that the release of chlorhexidine from composite resin reduced greatly after 5 days immersion in water, although continued slow release was observed for 20 days. $^{6}$

The sealants showed reduction in the compressive and DTS after addition of $1 \%$ chlorhexidine digluconate, which is not statistically significant. It is in accordance to the results. ${ }^{2-4}$ The reduction in the mechanical properties may be due to the following reasons: (A) incorporated $\mathrm{CHX}$ interfered with the binding of the filler and matrix phase, (B) the CHX caused disturbance in the curing of monomers, and $(\mathrm{C})$ release of the $\mathrm{CHX}$ could have caused a porous structure of the material. ${ }^{15,16}$ For the above reasons, Imatzo et al (2003) had suggested that addition of $\mathrm{CHX}$ was not appropriate for permanent restorations. ${ }^{15}$ Further studies are needed to evaluate the long-term effects of chlorhexidine addition on the mechanical properties of the sealants.

De Castilho et al (2013) investigated the in vitro and in vivo effects of addition of $1.25 \% \mathrm{CHX}$ to resin modified glass ionomer (RMGIC) cement. In vitro results revealed an improved antibacterial property without affecting the mechanical property of the cement. In vivo results after 3 months showed complete elimination of S. mutans from the cavities restored with RMGIC with $1.25 \%$ chlorhexidine. $^{5}$

The limitations of the current study include-a small sample size, the antibacterial activity was tested only for duration of 30 days, long-term effect on the mechanical properties of the sealants was not assessed, and chlorhexidine added as a powder could have yielded better results. Further in vivo studies are needed to prove the clinical efficacy of chlorhexidine addition to sealants.

Within the limitations of this pilot study it can be concluded that addition of $1 \%$ chlorhexidine to the glass ionomer and resin based sealants provided additional antibacterial activity to the sealant without significantly affecting the mechanical property of the sealant. Antibacterial property of the sealants lasting for 30 days makes the sealants more effective in preventing dental caries, thereby improving the quality of life of young children.

\section{CONCLUSION}

The addition of chlorhexidine in small concentrations provided antibacterial activity which can last for a period of 30 days without significantly altering the mechanical properties of the sealants. This can help the pediatric dentist give the child a better protection against caries and a promising healthy oral cavity.

\section{REFERENCES}

1. Beauchamp J, Caufield PW, Crall JJ, Donly KJ, Feigal R, Gooch B, Ismail A, Kohn W, Siegal M, Simonsen R, et al. Evidence-based clinical recommendations for the use of pit-and-fissure sealants: a report of the American Dental Association Council on Scientific Affairs. J Am Dent Assoc 2008;139(3):257-268.

2. Jedrychowski JR, Caputo AA, Kerper S. Antibacterial and mechanical properties of restorative materials combined with chlorhexidines. J Oral Rehabil 1983;10(5):373-381.

3. Deepalakshmi M, Poorni S, Miglani R, Rajamani I, Ramachandran S. Evaluation of the antibacterial and physical properties of glass ionomer cements containing chlorhexidine and cetrimide: an in vitro study. Ind J Dent Res 2010;21(4):552-556.

4. Turkun LS, Turkun M, Ertugrul F, Ateş M, Brugger S. Long-term antibacterial effects and physical properties of a chlorhexidine containing glass ionomer cement. J Esthet Restor Dent 2008;20(1):29-44.

5. de Castilho AR, Duque C, Negrini Tde C, Sacono NT, de Paula AB, de Souza Costa CA, Spolidório DM, PuppinRontani RM. In vitro and in vivo investigation of the biological and mechanical behaviour of resin-modified glass-ionomer-cement containing chlorhexidine. J Dent 2013;41(2):155-163.

6. Takemura K, Sakamoto Y, Staninec M, Kobayashi S, Suehiro K, Tsuchitani Y. Antibacterial activity of a bis-GMA based composite resin and antibacterial effect of chlorhexidine incorporation. Jpn J Conserv Dent 1983;26(4):540-547.

7. Bohannan HM. Caries distribution and the case for sealants. J Public Health Dent 1983;43(3):200-204.

8. Karlzen-Reuterving G, van Dijken JW. A 3-year follow-up of glass ionomer cement and resin fissure sealants. ASDC J Dent Child 1995;62(2):108-110.

9. Grewal N, Chopra R. The effect of fissure morphology and eruption time on penetration and adaptation of pit and 
fissure sealants: An SEM study. J Ind Soc Pedod Prev Dent 2008;26(2):59-63.

10. Hatibovic-Kofman S, Wright GZ, Braverman I. Microleakage of sealants after conventional, bur, and air abrasion preparation of pits and fissures. Pediatr Dent 1998;20(3):173-176.

11. McCue RW, McDougal FG, Shay DE. The antibacterial properties of some dental restorative materials. Oral Surg Oral Med Oral Pathol 1951 Sep;4(9):1180-1184.

12. Herrera M, Castillo A, Baca P, Carrion P. Antibacterial activity of glass-ionomer restorative cements exposed to cavity producing microorganisms. Oper Dent 1999;24(5):286-291.
13. Naorungroj S, Wei HH, Arnold RR, Swift EJ, Walter R. Antibacterial surface properties of fluoride containing resin based sealants. J Dent 2010;38(5):387-391.

14. Matalon S, Peretz B, Sidon R, Weiss EI, Slutzky H. Antibacterial properties of pit and fissure sealants combined with daily fluoride mouth rinse. Pediatr Dent 2010;32(1):9-13.

15. Imazato $S$. Antibacterial properties of resin composites and dentin bonding systems. Dent Mater 2003;19(6):449-457.

16. Addy M. In vitro studies into the use of denture base and soft liner materials as carriers for drugs in the mouth. J Oral Rehabil 1981;8(2):131-142. 